Research Paper

\title{
Quality of life and health status after Girdlestone resection arthroplasty in patients with an infected total hip prosthesis
}

\author{
Cornelis M. Vincenten ${ }^{1,3,4 凶}$, Brenda L. Den Oudsten ${ }^{2}$, Pieter K. Bos ${ }^{3}$, Stefan B.T. Bolder ${ }^{4}$, Taco Gosens ${ }^{1}$ \\ 1. Department of Orthopaedics, Elisabeth-Tweesteden Hospital, Tilburg, The Netherlands \\ 2. Department of Medical and Clinical Psychology, Tilburg University, Tilburg, The Netherlands \\ 3. Department of Orthopaedics, Erasmus MC, University Medical Center, Rotterdam, The Netherlands \\ 4. Department of Orthopaedics, Amphia Hospital, Breda, The Netherlands \\ $\triangle$ Corresponding author: Koen Vincenten, MD., Elisabeth-TweeSteden Hospital, Hilvarenbeekse weg 60, 5022GC, Tilburg, The Netherlands. \\ K.vincenten@etz.nl; Tel: +31 132210000 \\ (C) Ivyspring International Publisher. This is an open access article distributed under the terms of the Creative Commons Attribution (CC BY-NC) license \\ (https://creativecommons.org/licenses/by-nc/4.0/). See http://ivyspring.com/terms for full terms and conditions.
}

Received: 2018.07.09; Accepted: 2018.11.14; Published: 2019.01.01

\begin{abstract}
Introduction: The Girdlestone resection arthroplasty (GRA) is a salvage procedure for a recurrent or persistent prosthetic joint infection of the hip. This procedure negatively impacts the functional outcome and presumably also diminishes health status (HS) and quality of life (QOL). However, studies investigating the QOL after GRA are lacking. This cross-sectional study compares patients with a Girdlestone situation after an infected total hip prosthesis with a normative population with regard to HS and QOL.

Methods: Patients with a permanent GRA were suitable to be enrolled in the study. Subjects completed the World Health Organization Quality of life (WHOQOL-BREF) and the EuroQol 5 dimension 3 level version (EQ-5D-3L). Scores were compared with data from the normal population, from patients with a lower limb amputations and data from patients with a myocardial infarction.

Results: Sixty-three patients who underwent GRA between January 2000 and March 2017 completed the questionnaire. The median time between the GRA and competing the questionnaire was 48 months $(4-436)$. All WHOQOL-BREF domain scores were significantly lower in GRA patients compared to the normative data $(p<0.001)$, patients with myocardial infarction or lower limb amputation. EQ-5D-3L results showed that $\mathrm{HS}$ was significantly impaired in GRA patients when compared to normative data $(p<0.001)$ and also impaired when compared to data from lower limb amputations and myocardial infarctions.

Conclusion: HS and QOL scores in patients with a permanent Girdlestone situation after an infected hip prosthesis are significantly lower than Dutch normative scores. Patients with a permanent Girdlestone situation scored even lower on HS than patients with a lower limb amputation or a myocardial infarction.
\end{abstract}

Key words: Girdlestone, hip resection arthroplasty, quality of life, health status, hip

\section{Introduction}

Total hip replacement is one of the fastest growing orthopaedic procedures worldwide $(1,2)$. One of the leading causes for hip arthroplasty failure leading to Girdlestone Resection Arthroplasty (GRA) is a prosthetic joint infection (PJI) (3). PJI after total hip replacement is an increasing and severe complication $(2,4,5)$. GRA is defined as the removal of the hip prosthesis without a prosthetic replacement. The main objectives of GRA are to cure of infection and pain relief $(6,7)$. In most cases, GRA is part of the two-stage revision arthroplasty. In some cases of an infected total hip prosthesis with recurrent infection after reimplantation or no further reconstruction options, a GRA is considered as a last resort procedure (6). The situation that is created after GRA is termed the Girdlestone situation. The Girdlestone 
situation can result in a permanent clinical situation when 1) bone quality or soft tissue coverage is not strong enough to insert a new prosthesis, 2) the infection persists, or 3) when patients are unfit for surgery due to multiple comorbidities (8). Functional outcome after GRA is often impaired due to limb shortening, hip instability, pseudo arthrosis, and the inevitable need for a walking aid $(9,10)$. Two studies reviewed GRA after PJI and concluded that despite infection elimination and pain relief, more than 80 percent of the patients become functionally dependent because of their disabilities $(10,11)$. In the current literature, studies focusing on health status (HS) after GRA are scarce and remain inconclusive (7, 12). Moreover, studies focussing on quality of life (QOL) in patients after GRA are lacking. Studies on patient-reported outcomes measurements are important to evaluate the true impact of GRA in patients (13).

In this study, we will evaluate HS and QOL scores in patients with a Girdlestone situation after PJI. Furthermore, these results will be compared with normative data from a Dutch control population. In order to place the Girdlestone situation in a perspective of disease severity, we will also compare the scores obtained in the GRA population with data on HS in patients with a lower limb amputation and a myocardial infarction. Lower limb amputation was chosen because the patient loses (at least partly) a lower extremity function (hip/knee), which is comparable to GRA. Therefore, these groups may face similar consequences on HS and QOL. Myocardial infarction was chosen to bring the results in comparison to a medical perspective and because myocardial infarction is one of the most common diseases with a big impact on HS and QOL (14-17).

\section{Methods}

\section{Study design and setting}

A cross-sectional study was conducted between April and August 2017 from the Departments of Orthopaedics of the Elisabeth-Tweesteden Hospital in Tilburg and Waalwijk, the Erasmus MC, University Medical Centre in Rotterdam and the Amphia Hospital in Breda and Oosterhout. Additional patients were acquired through the Dutch Girdlestone Patient Association. This study was approved by the Noord-Brabant medical ethical committee (NW2017-17).

\section{Participants and procedure}

Patients were included when they underwent GRA between January 2000 and March 2017 and were 18 years of age or older. There was no upper age limit. All patients currently have a permanent Girdlestone situation. The definition that was used for a permanent Girdlestone situation is to have had a Girdlestone situation for $\geq 4$ months. This timeframe was chosen because the average prosthetic-free interval for the second stage revision is between 6-12 weeks (18).

Subjects were not eligible if they 1) received another indication for GRA than PJI (cerebral palsy, periprosthetic fracture, pathologic fracture, prosthetic loosening and hip dislocations), 2) had undergone a reimplantation at the time of the questionnaire or 3) had a reimplantation within 4 months. In addition, patients who were unable to complete the questionnaire (i.e., cognitive impairment or insufficient knowledge of the Dutch language) or were not being able to provide informed consent were not included into the study. When patients were eligible, one of the researchers (KV) approached them by phone explaining the purpose of this study and invited them to participate. We distributed an informative letter with a questionnaire to all patients by post to their home address. The questionnaire sets were sent and return between April and August 2017. All patients who gave written informed consent were able and willing to complete the questionnaire.

\section{Questionnaires}

The World Health Organization Quality of Life Brief Version (WHOQOL-BREF) measures QOL and contains 26 questions, divided into four domains and one facet. The domains consist of 1) Physical Health, 2) Psychological health, 3) Social Relationships, and 4) Environment. In addition, two items form the facet Overall QOL and general health. Items are rated on 5-point Likert scales. The mean score per domain ranges from 4 to 20 . A higher scores indicate a better QOL (19).

The EuroQoL 5 dimension 3 level version (EQ-5D-3L) questionnaire measures HS and comprises of two parts: the EQ-5D-3L self-classifier, a self-reported description of health problems according to a five-dimensional classification (i.e., mobility, self-care, usual activities, pain/discomfort and anxiety/depression) and is divided into 3 levels of perceived problems. Level 1 indicates no problems, level 2 indicates some problems and level 3 indicates extreme problems. The visual analogue scale (VAS) is designed to record the perceptions of a participant's current overall health state. The scale is graduated from 0 (the worst imaginable health state) to 100 (the best imaginable state). In both, the time frame is the day of responding. An index (EQ Index) based on the five dimensions to describe the overall HS of these patients. The EQ-5D-3L and WHOQOL-BREF has good to excellent psychometric properties of 
reliability (19-21). We used the Visual Analog Scale (VAS) for pain in rest and activity. Furthermore, the VAS for pain has acceptable psychometric properties (22). For the WHOQOL-BREF and EQ-5D-3L score Dutch norm scores and specified disease entities will be used to compare with our study population (20, 23).

Although HS and QOL are both patient-reported outcomes and multidimensional, the concepts differ. HS is merely an assessment of physical, psychological, and social functioning, whereas QOL focuses on the subjective evaluation of well-being in which the level of satisfaction is included $(19,24)$.

\section{Statistical analyses}

Descriptive analyses were performed to describe patient characteristics (i.e., age, gender, side, and duration of the Girdlestone situation). The Kolmogorov-Smirnov test was used to test the normality of study data. One sample T-tests were used to analyse whether the mean scores of the GRA group differs from the norm.

We performed a subgroup analysis for age, gender, and duration for the effect on QOL and HS. The significance level used in the analyses was $p<0.05$. Data analyses were conducted using SPSS version 23.

\section{Results}

\section{Patient cohort}

In total, 407 GRAs were recorded in the three participating hospitals; of these, in 206 patients a subsequent re-implantation was registered, of the remaining 201 cases, 65 died. In 32 cases, there was another indication than PJI for GRA. In 10 patients there was inability to complete the questionnaire due to dementia.

Ninety-four patients with GRA after failed PJI treatment were eligible for this study. Thirty-nine patients did not have up-to-date contact information or were not willing to participate in the current study. Eight patients were added through the Dutch Girdlestone Patient Association. In total, 63 patients were included in this study (Figure 1), of which 27 were males $(43 \%)$, the average age of patients was 71.7 years (SD 12.1), the Girdlestone situation was right-sided in $57 \%$ of the patients, the infection was cured in $61(97 \%)$ patients and the VAS score in rest and activity was $3.4 \quad(\mathrm{SD}=2.2)$ and $5.4 \quad(\mathrm{SD}=2.5)$ respectively (Table 1 ). The median time between the GRA and competing the questionnaire set was 48 (4 to 436) months. There was one patient from the Dutch Girdlestone association who had had a Girdlestone situation for 436 months. We choose to include this patient because the study population is already scarce.

Table 1. Patient characteristics of the study population $(n=63)$

\begin{tabular}{ll}
\hline & $\begin{array}{l}\text { Mean }(S D) \text { or } n(\%) \\
\text { patients }\end{array}$ \\
\hline Age, years (SD) & $71.8(12.1)$ \\
Male, $\mathrm{n}(\%)$ & $36(57)$ \\
Side, right, $\mathrm{n}(\%)$ & $36(57)$ \\
Mean VAS rest (SD) & $3.4(2.2)$ \\
Mean VAS activity (SD) & $5.4(2.5)$ \\
Median duration in months (minimum-maximum) & $48(4-436)$ \\
Cure of infection, $\mathrm{n}(\%)$ & $61(97)$
\end{tabular}

Abbreviations: VAS, visual analog scale, SD, standard deviation

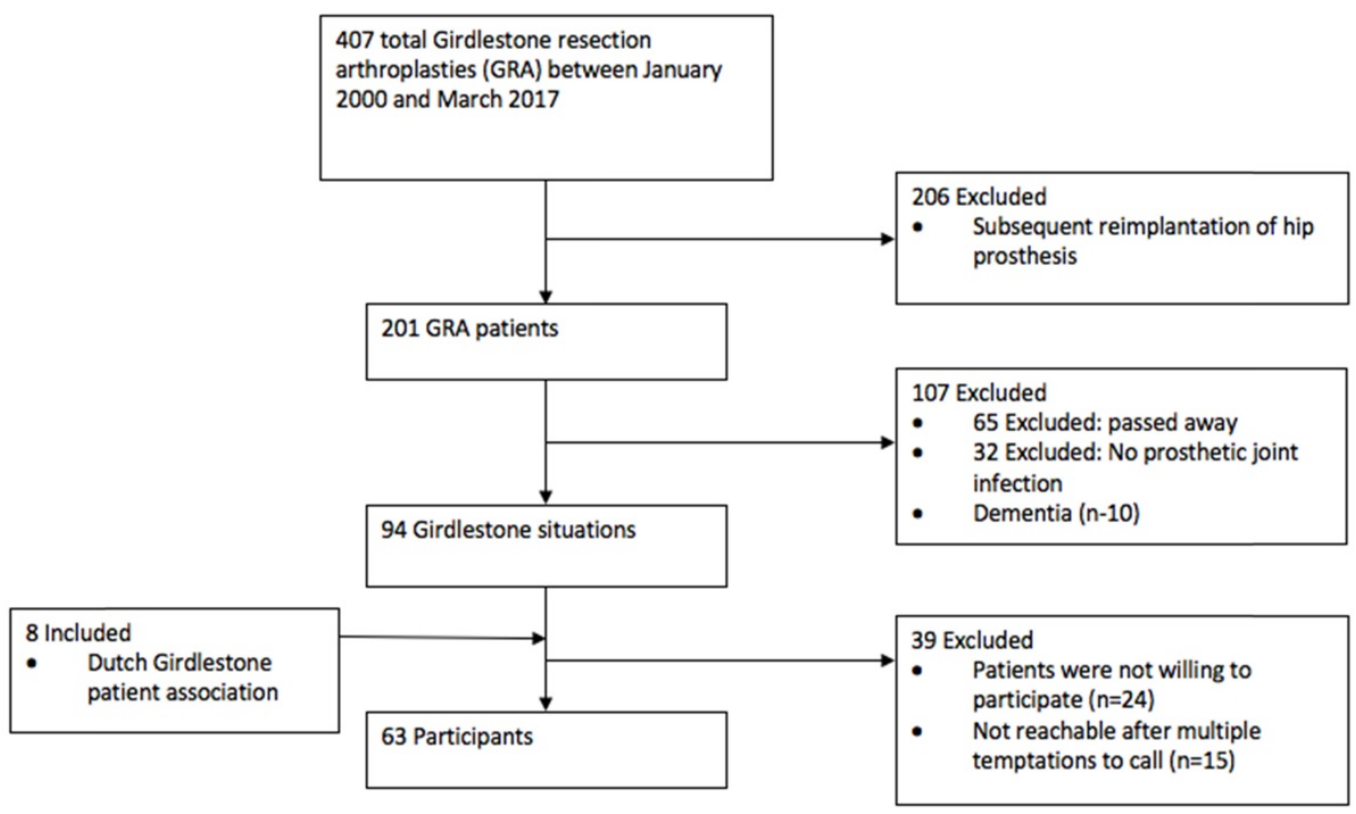

Figure 1. Flowchart showing inclusion/exclusion of the participants 


\section{Quality of life}

Means for the WHOQOL-BREF scores are presented in Table 2. Patients with a permanent situation after GRA scored significantly lower compared to the normative data in all domains $(p<0.001)$. When comparing GRA with normative data, the domain scores were respectively 11.4 vs. 18.3 for physical health, 12.9 vs. 16.6 for psychological health, 13.4 vs. 15.8 for social relationships and 13.1 vs. 15.9 for environment. When comparing the scores of the GRA population with patients with a lower limb amputation or a myocardial infarction, the Girdlestone group scored worse. The subgroup analysis for age, gender and duration for the effect on QOL and HS did not show any significant correlation.

Table 2. World Health Organization Quality of life (WHOQOL-BREF) facet and domain scores (range 4-20) compared with normative data and disease specific entities

\begin{tabular}{lllllll}
\hline Facet/domains & $\begin{array}{l}\text { Girdlestone } \\
\text { group } \\
\text { Mean (SD) }\end{array}$ & $\begin{array}{l}\text { Dutch } \\
\text { norm } \\
\text { Values }\end{array}$ & $\begin{array}{l}\text { Difference in } \\
\text { means (95\% } \\
\text { CI) }\end{array}$ & P & LLA $^{* 2}$ & MI $^{* 3}$ \\
\hline $\begin{array}{l}\text { Overall QOL and } \\
\text { General health }\end{array}$ & $11.3(1.8)$ & $14.2(2.4)$ & $2.9(2.2,3.8)$ & $<0.00114 .2$ & 14.2 \\
$\begin{array}{l}\text { Physical health } \\
\text { Psychological }\end{array}$ & $11.4(2.0)$ & $18.3(3.0)$ & $6.9(6.2,7.7)$ & $<0.00113 .0$ & 13.1 \\
$\begin{array}{l}\text { health } \\
\begin{array}{l}\text { Social } \\
\text { relationships }\end{array}\end{array}$ & $12.9(2.1)$ & $16.6(2.8)$ & $3.8(3.1,4.5)$ & $<0.00114 .4$ & 14.6 \\
Environment & $13.1(2.2)$ & $15.9(2.8)$ & $2.8(2.1,3.5)$ & $<0.00115 .6$ & 14.2 \\
\hline
\end{tabular}

Scores for each domain ranges from 4 to 20 . Higher scores denote higher quality of life.

Abbreviations: MI, myocardial infarction, LLA, lower limb amputation, CI, confidence interval, $\mathrm{SD}$, standard deviation.

*1 (20), *2(16), *3(14)

Table 3. Health status scores as assessed with the EQ-5D-3L domain compared to normative data and disease specific entities. Mean population EQ-VAS and mean EQ-5D-index. $(n=63)$

\begin{tabular}{|c|c|c|c|c|c|c|}
\hline Subscales & $\begin{array}{l}\text { Girdlestone } \\
\text { group (SD) }\end{array}$ & $\begin{array}{l}\text { Normative } \\
\text { data*(SD) }^{*}\end{array}$ & $\begin{array}{l}\text { Difference } \\
(95 \% \text { CI })\end{array}$ & P-value & LLA* & $\mathrm{MI}^{*}$ \\
\hline Mobility & $2.1(0.5)$ & $1.2(0.1)$ & $\begin{array}{l}-0.95(-0.95 \\
-0.93)\end{array}$ & $<0.001$ & 1.5 & 1.6 \\
\hline Self-care & $2.0(0.7)$ & $1.0(0.1)$ & $\begin{array}{l}-0.96(-0.99 \\
-0.93)\end{array}$ & $<0.001$ & 1.8 & 1.2 \\
\hline Usual activities & $2.3(0.7)$ & $1.2(0.1)$ & $\begin{array}{l}1.13(-0.99 \\
-0.93)\end{array}$ & $<0.001$ & 2.0 & 1.5 \\
\hline Pain/discomfort & $2.0(0.6)$ & $1.4(0.2)$ & $\begin{array}{l}0.63(-1.00, \\
-0.92)\end{array}$ & $<0.001$ & 1.8 & 1.7 \\
\hline Anxiety/depression & $1.7(0.7)$ & $1.2(0.1)$ & $\begin{array}{l}0.51(0.48, \\
0.55)\end{array}$ & $<0.001$ & 1.6 & 1.4 \\
\hline EQ-VAS & $52.4(18.9)$ & 82 (11.6) & $\begin{array}{l}29.60(26.71 \\
-32.05)\end{array}$ & $<0.001$ & & \\
\hline EQ-5D index & $0.4(0.3)$ & $0.9(0.1)$ & $\begin{array}{l}0.50 \\
(0.47-0.53)\end{array}$ & $<0.001$ & & \\
\hline
\end{tabular}

$1=$ no problems, $2=$ some problems, $3=$ extreme problems, EQ-VAS $(0-100)$, EQ-5D index (0-1).

Abbreviations: MI, myocardial infarction, LLA, lower limb amputation, CI, confidence interval, SD, standard deviation.

*(14, 17, 23)

\section{Health status}

GRA respondents scored significantly lower on all subscale scores of the EQ-5D-3L compared to data of the normal population (Table 3). Scores (a higher score means more problems in that specific subscale) were significantly different in the subscales of mobility (2.1 vs 1.2), self-care (2.0 vs 1.0$)$, usual activities: (2.3 vs 1.2) pain/discomfort; 2.0 vs 1.4 , anxiety/depression; 1.7 vs 1.2 (Table 3 ). These results show that HS is significantly impaired in GRA patients compared to normative data $(\mathrm{p}<0.001)$. Of the patients with GRA, $95.6 \%$ experienced problems in mobility, $77.8 \%$ in self-care, $91.1 \%$ in daily activities, $84.5 \%$ had pain and discomfort, and $55.6 \%$ had symptoms of anxiety or depression. The average self-reported health scale (EQ-VAS) for GRA patients and normative data was respectively 52.4 (32.4 to 70.2) and 82 (70.4 to 93.6).

\section{Discussion}

In this study, we assessed HS and QOL in patients who underwent GRA after an infected total hip replacement. Patients with GRA after an infected total hip prosthesis have a reduced HS and QOL scores compared with the normal population and patients with lower limb amputation and myocardial infarction (14-17). QOL was significantly lower in all domains of both questionnaires. Even when comparing them with lower limb amputations and myocardial infarctions, it showed impaired results in all of them.

Although GRA has proved to be a successful method for eradication of infection, it comes at a high expense due to the low functional outcome of the procedure. First, GRA leads to pseudo-artrosis with a leg length discrepancy, muscle weakness and a decreased range of motion (6-9, 11, 25, 26). Additionally, patients that undergo GRA are generally not deemed fit for a single- or two-stage revision of a total hip replacement due to their age and multiple comorbidities. As this patient group already has significant functional disabilities, a GRA procedure will undoubtedly lead to a critical situation that makes patients functionally dependent in their daily activities $(8,11,27)$. As they are less ambulant which may result in a restricted freedom of maintaining the daily lifestyle, influencing their psychological wellbeing. It has been shown that a strong social network may relieve stress in crisis situations, as after a GRA. Social support may work as a protective factor and thus may increase QOL. Therefore, more emphasis on the expectation and social management of the postoperative situation could enhance the perceived QOL in this specific population $(28,29)$.

Two previous studies that measured HS based on limited groups of patients provided inconclusive results using the short-form health survey to measure 
HS $(7,12)$. Barbaric et al. retrospectively compared 53 patients having a Girdlestone situation with 20 patients who underwent revision arthroplasty. The average SF-36 physical component summary and mental component summary were respectively 27.7 and 45.5, which is low. Yamamoto et al. retrospectively reported in 9 patients the SF-36 score with a unilateral Girdlestone situation. Measuring an overall health status of 75.4 which is a relatively high score (range 0-100). Thus, both studies where not in line with each other. The relatively high score in Yamamoto et al can be explained by the number of patients which is too low to draw firm conclusions.

This study shows that patients have lower HS scores compared to the norm population with an EQ-5D-3L index score of 0.4. Similar to previous studies, the lowest scores were measured in functional subscales: mobility, usual activities, self-care, pain/discomfort $(7,12)$. The low EQ-VAS suggests low general health conditions of the included patients and corresponds to previous studies $(10,11)$.

Helwig et al. collected data of patients with a prosthetic joint infection (hip or knee) and measured the overall QOL using the short form health survey 12 (SF-12) and compare the successful treatment with the ongoing PJI. Their results showed that QOL is substantially reduced after a PJI when not treated successfully(30). This shows that already in the process from PJI to GRA the QOL is impaired.

The high pain score observed in our study population was not in line with previous studies $(6,8$, 11) and questions the reported pain relief of GRA. In the previous studies pain was scored by other measurements than VAS pain score $(6,8-11,25,26$, $31)$. Therefore, the distinction in pain score can partially be explained by the differentiation between the rest and activity VAS pain score we were using.

This study has included the highest number of patients with a permanent Girdlestone situation, with an average duration of 57.9 months for having a Girdlestone situation. Patients who are offered GRA were mainly elderly with an average age of 72 years, which is in line with other studies $(7,8,26)$.

When patients have a temporary Girdlestone situation, they will be getting a reimplantation in the near future (second-stage revision). Their perception on QOL may be more positive because of their future perspectives.

As the patients that underwent GRA is a heterogeneous group, with different indication to undergo a GRA, various combinations of comorbidities, and a high age, it remains difficult to extrapolate the results to all patients that underwent a GRA (32). A combination of the functional evaluation and measuring patient-reported outcomes both pre-and postoperatively would truly measure the impact of a GRA on both objective and subjective scores. Information on comorbidities is lacking in this study, but could have a significant influence on the outcome of our study $(11,12,33)$. Including patients through patient groups could introduce bias to our study population as they could have various reasons for encouragement to enroll into this study, for example due to dissatisfaction of their individual functional outcome.

In conclusion, patients with a Girdlestone situation after an infected total hip prosthesis have a significantly impaired HS and QOL comparing to the normal population and other disease specific entities like lower limb amputation and myocardial infarction. They experience a high level of pain in rest and during activity. The importance of informing the patient on their postoperative status and the management of expectations needs to be underlined in a proper setting. We believe that this study offers valuable information when it comes to $\mathrm{HS}$ and QOL in patients after GRA. As the clinical situation after GRA highly restricts patients to functioning in daily activities, withholds the possibility to be part of a social network and greatly diminishes their psychological wellbeing, we stress the importance to aim to prevent a permanent Girdlestone situation and consider reimplantation if possible.

\section{Abbreviations}

GRA: Girdlestone Resection Arthroplasty

WHOQOL-BREF: World Health Organization Quality Of Life Scale Brief Version

QOL: Quality Of Life

HS: Health Status

PJI: Prosthetic Joint Infection

EQ-5D-3L: The EuroQol 5 Dimension 3 Level Version

\section{Acknowledgements}

We thank S. Morrenhof-Atkinson for correction of the English language.

\section{Competing Interests}

The authors have declared that no competing interest exists.

\section{References}

1. Learmonth ID, Young C, Rorabeck C. The operation of the century: total hip replacement. Lancet (London, England). 2007;370(9597):1508-19.

2. Lenguerrand E, Whitehouse MR, Beswick AD, Jones SA, Porter ML, Blom AW. Revision for prosthetic joint infection following hip arthroplasty: Evidence from the National Joint Registry. Bone \& joint research. 2017;6(6):391-8.

3. Liodakis E, Antoniou J, Zukor DJ, Huk OL, Epure LM, Bergeron SG. Major Complications and Transfusion Rates After Hemiarthroplasty and Total Hip Arthroplasty for Femoral Neck Fractures. J Arthroplasty. 2016;31(9):2008-12.

4. Dutch arthroplasty register (LROI), annual report 2016.

5. Buttaro MA, Zanotti G, Comba FM, Piccaluga F. Primary Total Hip Arthroplasty With Fourth-Generation Ceramic-on-Ceramic: Analysis of 
Complications in 939 Consecutive Cases Followed for 2-10 Years. J Arthroplasty. 2017;32(2):480-6.

6. Castellanos J, Flores X, Llusa M, Chiriboga C, Navarro A. The Girdlestone pseudarthrosis in the treatment of infected hip replacements. Int Orthop. 1998;22(3):178-81.

7. Yamamoto PA, Lahoz GL, Takata ET, Masiero D, Chamlian TR. Avaliação da função e qualidade de vida em pacientes submetidos a artroplastia de ressecção tipo Girdlestone. Acta Ortopédica Brasileira. 2007;15:214-7.

8. Sharma H, De Leeuw J, Rowley DI. Girdlestone resection arthroplasty following failed surgical procedures. Int Orthop. 2005;29(2):92-5.

9. Bourne RB, Hunter GA, Rorabeck CH, Macnab JJ. A six-year follow-up of infected total hip replacements managed by Girdlestone's arthroplasty. J Bone Joint Surg Br. 1984;66(3):340-3.

10. Sharma H, Kakar R. Outcome of Girdlestone's resection arthroplasty following complications of proximal femoral fractures. Acta Orthop Belg. 2006;72(5):555-9.

11. Basu I, Howes M, Jowett C, Levack B. Girdlestones excision arthroplasty: current update. Int J Surg. 2011;9(4):310-3.

12. Barbaric K, Aljinovic A, Dubravcic ID, Delimar D, Bicanic G. Patient satisfaction after revision hip arthroplasty or resection hip arthroplasty due to periprosthetic infection. Collegium antropologicum. 2014;38(2):605-10.

13. Conrad P, Barker KK. The social construction of illness: key insights and policy implications. Journal of health and social behavior. 2010;51 Suppl:S67-79.

14. Briggs AH, Bhatt DL, Scirica BM, Raz I, Johnston KM, Szabo SM, et al. Health-related quality-of-life implications of cardiovascular events in individuals with type 2 diabetes mellitus: A subanalysis from the Saxagliptin Assessment of Vascular Outcomes Recorded in Patients with Diabetes Mellitus (SAVOR)-TIMI 53 trial. Diabetes Res Clin Pract. 2017;130:24-33.

15. Deans SA, McFadyen AK, Rowe PJ. Physical activity and quality of life: A study of a lower-limb amputee population. Prosthetics and orthotics international. 2008;32(2):186-200.

16. Sarah AD, Angus KM, Philip JR. Physical activity and quality of life: A study of a lower-limb amputee population. Prosthetics and Orthotics International. 2008;32(2):186-200.

17. Godlwana L, Stewart A, Musenga E. Quality of life following a major lower limb ampu tation in Johann esburg, South Africa. South African Journal of Physiotherapy. 2012;68(2):17-22

18. Cooper HJ, Della Valle CJ. The two-stage standard in revision total hip replacement. The bone \& joint journal. 2013;95-b(11 Suppl A):84-7.

19. Group W. Development of the World Health Organization WHOQOL-BREF quality of life assessment. Psychological medicine. 1998;28(3):551-8.

20. Skevington SM, Lotfy M, O'Connell KA, Group W. The World Health Organization's WHOQOL-BREF quality of life assessment: psychometric properties and results of the international field trial. A report from the WHOQOL group. Qual Life Res. 2004;13(2):299-310.

21. Yfantopoulos JN, Chantzaras AE. Validation and comparison of the psychometric properties of the EQ-5D-3L and EQ-5D-5L instruments in Greece. The European journal of health economics : HEPAC : health economics in prevention and care. 2017;18(4):519-31.

22. McCormack HM, Horne DJ, Sheather S. Clinical applications of visual analogue scales: a critical review. Psychol Med. 1988;18(4):1007-19.

23. Mangen MJ, Bolkenbaas M, Huijts SM, van Werkhoven CH, Bonten MJ, de Wit GA. Quality of life in community-dwelling Dutch elderly measured by EQ-5D-3L. Health Oual Life Outcomes. 2017;15(1):3.

24. Group W. Development of the WHOQOL: Rationale and current status. International Journal of Mental Health. 1994;23(3):24-56.

25. de Laat EA, van der List JJ, van Horn JR, Slooff TJ. Girdlestone's pseudarthrosis after removal of a total hip prosthesis; a retrospective study of 40 patients. Acta Orthop Belg. 1991;57(2):109-13.

26. Vatopoulos PK, Diacomopoulos GJ, Demiris CS, Gorgolis J, Papathanassiou BT. Girdlestone's operation: a follow-up study. Acta orthopaedica Scandinavica. 1976;47(3):324-8.

27. Mahmoud SS, Sukeik M, Alazzawi S, Shaath M, Sabri O. Salvage Procedures for Management of Prosthetic Joint Infection After Hip and Knee Replacements. The open orthopaedics journal. 2016;10:600-14.

28. Umberson D, Montez JK. Social relationships and health: a flashpoint for health policy. Journal of health and social behavior. 2010;51 Suppl:S54-66.

29. Lemos CF, Rodrigues MP, Veiga JRP. Family income is associated with quality of life in patients with chronic kidney disease in the pre-dialysis phase: a cross sectional study. Health and Quality of Life Outcomes. 2015;13(1):202.

30. Helwig P, Morlock J, Oberst M, Hauschild O, Hübner J, Borde J, et al. Periprosthetic joint infection-effect on quality of life. Int Orthop. 2014;38(5):1077-81

31. Carlsson AM. Assessment of chronic pain. I. Aspects of the reliability and validity of the visual analogue scale. Pain. 1983;16(1):87-101.

32. Leung L. Validity, reliability, and generalizability in qualitative research. Journal of Family Medicine and Primary Care. 2015;4(3):324-7.

33. Megari K. Quality of Life in Chronic Disease Patients. Health Psychol Res. 2013;1(3):e27. 